\title{
On an Asymptotical Moments Problem
}

\author{
Slavko Simic ${ }^{*}$
}

Mathematical Institute SANU, Kneza Mihaila 36, 11000 Belgrade, Serbia

Abstract: In this article we shall give some necessary and sufficient conditions for a discrete law $F$ such that the asymptotic equivalence

$$
E X_{n}^{\mu} \sim\left(E X_{n}\right)^{\mu}\left(E X_{n} \rightarrow \infty\right)
$$

takes place for all positive $\mu$. An extension to the class of regularly varying moments is also given.

\section{INTRODUCTION}

For a sequence of random variables $\left(X_{n}\right)$ define a discrete probability law $F$ by

$$
P\left\{X_{n}=k\right\}=p_{n k} \geq 0, \quad k=0,1,2, \cdots, n ; \quad \sum_{k=0}^{n} p_{n k}=1 .
$$

Define as usual the expectation $E X_{n}$ and variance $\sigma^{2} X_{n}$ as

$$
E X_{n}:=\sum_{k=1}^{n} k p_{n k} ; \quad \sigma^{2} X_{n}:=E X_{n}^{2}-\left(E X_{n}\right)^{2}
$$

moments of the $m$-th order are

$$
E X_{n}^{m}:=\sum k^{m} p_{n k}, \quad m=2,3, \cdots .
$$

The question of moments convergence is a difficult one and entirely depends on the characteristics of the law $F$.

But if

$$
E X_{n} \rightarrow \infty(n \rightarrow \infty)
$$

then, due to Jensen's inequality, all other moments are also unbounded and there is the problem of their asymptotic evaluation.

In this paper we shall give some conditions such that the asymptotic equivalence

$$
E X_{n}^{\mu} \sim\left(E X_{n}\right)^{\mu}(n \rightarrow \infty)
$$

holds for all real $\mu>0$, whenever $E X_{n} \rightarrow \infty(n \rightarrow \infty)$.

\section{RESULTS}

It turns out that, under a specific conditions, the validity of the asymptotic relation (1) for some $\mu=m>1$, implies its validity for all moments of lesser order. This result is characterized in the first proposition.

*Address correspondence to this author at the Mathematical Institute SANU, Kneza Mihaila 36, 11000 Belgrade, Serbia;

E-mail: ssimic@turing.mi.sanu.ac.yu

2000 Mathematics Subject Classification 60E05
Proposition 1. Let $m>1$ and $E X_{n} \rightarrow \infty(n \rightarrow \infty)$; then the asymptotic equivalence

$$
E X_{n}^{\mu} \sim\left(E X_{n}\right)^{\mu}
$$

holds for each real $\mu, 0<\mu \leq m$, if and only if

$$
\limsup _{n \rightarrow \infty} \frac{E X_{n}^{m}}{\left(E X_{n}\right)^{m}} \leq 1
$$

Another quite unexpected result is the following

Proposition 2. If $E X_{n} \rightarrow \infty$ and the probability generating function $E S^{X}$,

$$
E s^{X_{n}}:=\sum_{k=0}^{n} p_{n k} s^{k},
$$

belongs to the class $\mathcal{H}$ of Hurwitz polynomials, then the relation

$$
E X_{n}^{\mu} \sim\left(E X_{n}\right)^{\mu}(n \rightarrow \infty)
$$

holds for each $\mu>0$.

Recall that the class $\mathcal{H}$ consists of all polynomials with non-negative coefficients whose zeros lie entirely in the left complex half-plane (including imaginary axes). This class is of importance in Mechanics and the Theory of Dynamic Stability [1].

Another statement concerning Hurwitz polynomials follows

Proposition 3. Let $H_{n}(c):=\sum_{k=0}^{n} a_{n k} c^{k}, H_{n}(c) \in \mathcal{H}$.

For a sequence of random variables $\left(Y_{n}\right)$ define the probability law $F_{H}$, with a parameter $c>0$, as

$P_{H}\left\{Y_{n}=k\right\}:=\frac{a_{n k} c^{k}}{H_{n}(c)}, \mathrm{k}=0,1, \cdots, n$

If, for some $c>0, E_{H} Y_{n}=\frac{c H_{n}^{\prime}(c)}{H_{n}(c)} \rightarrow \infty \quad(n \rightarrow \infty)$, then

$$
E_{H} Y_{n}^{\mu} \sim\left(E_{H} Y_{n}\right)^{\mu} \quad(\mathrm{n} \rightarrow \infty)
$$


for each $\mu>0$.

Further generalization leads to the concept of regularly varying moments [2, p. 335].

It is said that the function $\ell$ is slowly varying in Karamata's sense if it is positive, continuous and satisfies $\ell(\lambda x) \sim \ell(x),(x \rightarrow \infty)$ for each $\lambda>0[2$, p. 6].

Some examples of slowly varying functions are

$1,(\log x)^{a},(\log \log x)^{b}, \exp \left((\log x)^{c}\right) ; a, b \in R, 0<c<1$.

The expression

$$
E X_{n}^{\rho} \ell\left(X_{n}\right):=\sum_{k=1}^{n} k^{\rho} \ell(k) p_{n k}
$$

is called regularly varying moment of order $\rho$.

For asymptotic behavior of those moments we have

Proposition 4. If

(i) $1 / E X_{n}=O(1 / n) ; \quad(i i) \sigma^{2}\left(X_{n}\right)=o\left(n^{2}\right) \quad(n \rightarrow \infty)$,

then the asymptotic relation

$$
E X_{n}^{\rho} \ell\left(X_{n}\right) \sim\left(E X_{n}\right)^{\rho} \ell\left(E X_{n}\right) \quad(n \rightarrow \infty),
$$

holds for each $\rho>0$ and every slowly varying function $\ell(\cdot)$.

\section{PROOFS}

It is obvious that the condition (2) is necessary for $E X_{n}^{m}$ $\sim\left(E X_{n}\right)^{m}$ to hold. We shall prove that it is also sufficient for the statement of Proposition 1.

Indeed, since $m>1$, by Jensen's inequality [3, p. 104] it follows that

Therefore by (2),

$$
E X_{n}^{m} \geq\left(E X_{n}\right)^{m}
$$

$$
1 \leq \liminf _{n \rightarrow \infty} \frac{E X_{n}^{m}}{\left(E X_{n}\right)^{m}} \leq \limsup _{n \rightarrow \infty} \frac{E X_{n}^{m}}{\left(E X_{n}\right)^{m}} \leq 1 .
$$

i. e.,

$$
E X_{n}^{m} \sim\left(E X_{n}\right)^{m} \quad(n \rightarrow \infty) .
$$

Now we shall prove the case $0<\mu<m$. For this purpose we need well-known Liapunov moments inequality [3, p. $110]$.

Lemma 1. For $0<a<b<c$, we have

$\left(E X_{n}^{b}\right)^{c-a} \leq\left(E X_{n}^{a}\right)^{c-b}\left(E X_{n}^{c}\right)^{b-a}$.

Applying (4) for $1<\mu<m$, we get

$$
\left(E X_{n}^{\mu}\right)^{m-1} \leq\left(E X_{n}\right)^{m-\mu}\left(E X_{n}^{m}\right)^{\mu-1} .
$$

By this and Jensen's inequality we obtain

$1 \leq \frac{E X_{n}^{\mu}}{\left(E X_{n}\right)^{\mu}} \leq\left(\frac{E X_{n}^{m}}{\left(E X_{n}\right)^{m}}\right)^{\frac{\mu-1}{m-1}}$

For $0<\mu<1$, Jensen's inequality gives

$$
E X_{n}^{\mu} \leq\left(E X_{n}\right)^{\mu} .
$$

Combining this with (4), $(\mathrm{a}=\mu, \mathrm{b}=1, \mathrm{c}=\mathrm{m})$ we get

$1 \geq \frac{E X_{n}^{\mu}}{\left(E X_{n}\right)^{\mu}} \geq\left(\frac{E X_{n}^{m}}{\left(E X_{n}\right)^{m}}\right)^{\frac{-(1-\mu)}{m-1}}$

Hence, letting $n \rightarrow \infty$, by (3), (5) and (6) we obtain the assertion of Proposition 1.

\section{Proof of Proposition 2}

This is a consequence of the assertion from Proposition 3 below, with

$$
c:=1 ; a_{n k} / H_{n}(1):=p_{n k} .
$$

\section{Proof of Proposition 3}

In the sequel we shall need the following

Lemma 2. For any $H \in \mathcal{H}$ and thus induced law $F_{H}$, we have

$$
0 \leq \sigma_{H}^{2}\left(Y_{n}\right)<2 E_{H}\left(Y_{n}\right) .
$$

Proof: From the basic algebra theorems and definition of Hurwitz polynomials, we get the representation

$$
H(c)=H_{n}(c)=a_{n n} \prod_{k \leq n}\left(c+z_{n k}\right), \quad a_{n n}>0, \quad \Re z_{n k} \geq 0 .
$$

Hence,

$$
E_{H} Y_{n}=\sum_{k \leq n} k a_{n k} c^{k} / H(c)=\frac{c H^{\prime}(c)}{H(c)}=\sum_{k \leq n} \frac{c}{c+z_{n k}}=\sum_{k \leq n} u_{n k}=\sum_{k \leq n} \Re u_{n k},
$$

and

$$
\sigma_{H}^{2}\left(Y_{n}\right)=c \frac{d}{d c}\left(E_{H} Y_{n}\right)=\sum_{k \leq n} \frac{c z_{n k}}{\left(c+z_{n k}\right)^{2}}=\sum_{k \leq n} v_{n k}=\sum_{k \leq n} \Re v_{n k},
$$

Since c $>0, \mathfrak{R} z_{n k} \geq 0$, we get

$$
0 \leq \frac{\mathfrak{N} v_{n k}}{\Re u_{n k}}=\frac{\mathfrak{N} z_{n k}\left(c+\mathfrak{N} z_{n k}\right)^{2}+\left(\mathfrak{I} z_{n k}\right)^{2}\left(2 c+\mathfrak{N} z_{n k}\right)}{\left(c+\mathfrak{R} z_{n k}\right)\left(\left(c+\mathfrak{N} z_{n k}\right)^{2}+\left(\mathfrak{I} z_{n k}\right)^{2}\right)}<2 .
$$

Therefore,

$$
\sigma_{H}^{2}\left(Y_{n}\right)=\sum_{k \leq n} \Re v_{n k}=\sum_{k \leq n} \Re u_{n k}\left(\frac{\Re v_{n k}}{\Re u_{n k}}\right)<2 \sum_{k \leq n} \Re u_{n k}=2 E_{H} Y_{n},
$$

and the proof is done.

Let us consider now a sequence of polynomials $\left(H_{m}(c)\right), m$ $=1,2, \cdots$ given by the recurrence relation

$$
\mathrm{H}_{\mathrm{m}}(\mathrm{c})=c H_{m-1}^{\prime}(c), \quad H_{0}(c)=H(c) .
$$

We have

Lemma 3. For each $m \in N, \quad H_{m}(c) \in \mathcal{H}$.

Proof: Suppose that $H_{m-1}(\mathrm{c}) \in \mathcal{H}$. By well-known Gauss Theorem, the zeros of $H_{m-1}(c)$ are not away from the convex polygon enveloping the zeros of $H_{m-1}(c)$. Therefore, all zeros of $c H^{\prime}(c)$ also belong to the left complex halfplane i. e. $H_{m}(c) \in \mathcal{H}$. Since $H_{0}(c)=H(c) \in \mathcal{H}$, the proof follows by induction in $m$. 
Because

$$
H_{m}(c)=\sum_{k \leq n} k^{m} a_{n k} c^{k}, \quad m \in N
$$

we get

$$
\text { (i) } E_{H_{m}} Y_{n}=\frac{H_{m+1}(c)}{H_{m}(c)} ;(i i) \sigma_{H_{m}}^{2}\left(Y_{n}\right)=E_{H_{m+1}} Y_{n} \cdot E_{H_{m}} Y_{n}-\left(E_{H_{m}} Y_{n}\right)^{2}
$$

Combining (ii) with Lemmas 2 and 3, we obtain

$$
E_{H_{m+1}} Y_{n}-E_{H_{m}} Y_{n}=\sigma_{H_{m}}^{2}\left(Y_{n}\right) / E_{H_{m}} Y_{n}<2,
$$

i. e. by summing

$$
E_{H_{m}} Y_{n}<E_{H} Y_{n}+2 m, \quad m=1,2, \cdots
$$

Now, from (i) and (7), it follows that

$$
E_{H} Y_{n}^{m}=\frac{H_{m}(c)}{H(c)}=\prod_{k=0}^{m-1} E_{H_{k}} Y_{n}<\prod_{k=0}^{m-1}\left(E_{H} Y_{n}+2 k\right)
$$

Since by assumption $E_{H} Y_{n} \rightarrow \infty(n \rightarrow \infty)$, this gives

$$
\limsup _{n \rightarrow \infty} \frac{E_{H} Y_{n}^{m}}{\left(E_{H} Y_{n}\right)^{m}} \leq 1
$$

Hence, by Proposition 1 the assertion from Proposition 3 follows.

\section{Proof of Proposition 4}

Note that the conditions ( $i$ ) and (ii) imply

$$
E X_{n} \rightarrow \infty(n \rightarrow \infty),
$$

and

$$
\begin{gathered}
\sigma^{2}\left(X_{n}\right)=E X_{n}^{2}-\left(E X_{n}\right)^{2}=o\left(n^{2}\right)=\left(n / E X_{n}\right)^{2} o\left(\left(E X_{n}\right)^{2}\right) \\
=O(1) o\left(\left(E X_{n}\right)^{2}\right)=o\left(\left(E X_{n}\right)^{2}\right) .
\end{gathered}
$$

Hence

$$
E X_{n}^{2} \sim\left(E X_{n}\right)^{2} \quad(n \rightarrow \infty)
$$

Also,

i. e.,

$E\left|X_{n}-E X_{n}\right|=o(n) \quad(n \rightarrow \infty)$.

We consider firstly the case $\ell(x)=1, \rho \in N$.

Let $q>2, q \in N$. We have

$$
\begin{gathered}
0 \leq \psi_{n, q}:=E X_{n}^{q}-E X_{n} E X_{n}^{q-1} \\
=\sum_{k \leq n} k^{q-1} p_{n k}\left(k-E X_{n}\right) \leq n^{q-1} E\left|X_{n}-E X_{n}\right| .
\end{gathered}
$$

Applying the condition ( $i$ ) and (8), we get

$\frac{\psi_{n, q}}{\left(E X_{n}\right)^{q}}=o(1)\left(n / E X_{n}\right)^{q}=o(1) O(1)=o(1) \quad(n \rightarrow \infty)$.

Finally, by the triangle inequality it follows

$$
\left|\frac{E X_{n}^{q}}{\left(E X_{n}\right)^{q}}-1\right| \leq\left|\frac{E X_{n}^{q-1}}{\left(E X_{n}\right)^{q-1}}-1\right|+\frac{\psi_{n, q}}{\left(E X_{n}\right)^{q}},
$$

and the proof can be carried out by induction in $q$.

Since $q>2$ is arbitrary, by Proposition 1 it follows that the proof is valid for all $\rho>0$ and $\ell(x)=1$. For the general case we need the next

Lemma 4. If the matrix $\left\{A_{n k}\right\}$ satisfies

$$
\text { (i) } \sum_{k \leq n} A_{n k} \rightarrow 1 ; \quad \text { (ii) } \sum_{k \leq n} k^{-v}\left|A_{n k}\right|=O\left(n^{-v}\right) \quad(n \rightarrow \infty)
$$

with some $v>0$, then

$$
\sum_{k \leq n} A_{n k} \ell(k) \sim \ell(n) \quad(n \rightarrow \infty)
$$

for each slowly varying $\ell(\cdot)(\operatorname{cf}[4])$.

Putting $A_{n k}:=k^{\rho} p_{n k} / E X_{n}^{\rho} ; v:=\rho / 2$, we get

$$
\begin{aligned}
& \sum_{k \leq n} A_{n k}=1 ; \\
& \sum_{k \leq n} k^{-v} A_{n k}=E X_{n}^{\rho / 2} / E X_{n}^{\rho} \sim\left(E X_{n}\right)^{-v}=O\left(n^{-v}\right) \quad(n \rightarrow \infty)
\end{aligned}
$$

Therefore, by Lemma 4 we have

$$
\sum_{k \leq n}\left(k / E X_{n}\right)^{\rho} \ell(k) p_{n k} \sim \ell(n) \quad(n \rightarrow \infty) .
$$

Since $1 \leq n / E X_{n}=O(1)(n \rightarrow \infty)$, the Uniform Convergence Theorem [2, p. 6] gives $\ell(n) \sim \ell\left(E X_{n}\right)$, and we finally obtain

$$
E X_{n}^{\rho} \ell\left(X_{n}\right):=\sum_{k \leq n} k^{\rho} \ell(k) p_{n k} \sim\left(E X_{n}\right)^{\rho} \ell\left(E X_{n}\right) \quad(n \rightarrow \infty) .
$$

\section{EXAMPLES}

We shall apply our results on some well-known probability laws. Let us to consider firstly the Gaussian Hypergeometric Law given by

$$
P\left\{X_{n}=k\right\}=\left(\begin{array}{c}
M \\
k
\end{array}\right)\left(\begin{array}{c}
N-M \\
n-k
\end{array}\right) /\left(\begin{array}{c}
N \\
k
\end{array}\right) .
$$

Choosing parameters $M$ and $N$ such that

$$
N=2 M=2 n+2 A, \quad A \geq 0,
$$

we obtain that the probability generating function $E s^{X}$,

$$
E s^{X_{n}}=\left(\begin{array}{c}
2 n+2 A \\
n
\end{array}\right)^{-1} \sum_{k}\left(\begin{array}{c}
n+A \\
k
\end{array}\right)\left(\begin{array}{c}
n+A \\
n-k
\end{array}\right) s^{k} .
$$

is from the class of ultraspherical polynomials [5, pp. 8186].

Because of orthogonality, all their zeros are real and negative i. e.

$$
E S^{X} \in \mathcal{H} \text {. }
$$

Since $E X_{n}=n / 2$, it follows that we can use Proposition 2 to determine asymptotic behavior of all moments of positive order.

Much stronger result can be obtained if we notice that in this case [3]

$$
\sigma^{2}\left(X_{n}\right)=\frac{n(n+2 A)}{4(2 n+2 A-1)}=o\left(n^{2}\right) \quad(n \rightarrow \infty) .
$$


Therefore, applying Proposition 4, we obtain asymptotic behavior of regularly varying moments

$$
\begin{aligned}
& E X_{n}^{\rho} \ell\left(X_{n}\right):=\left(\begin{array}{c}
2 n+2 A \\
n
\end{array}\right)^{-1} \sum_{k} k^{\rho} \ell(k)\left(\begin{array}{c}
n+A \\
k
\end{array}\right)\left(\begin{array}{c}
n+A \\
n-k
\end{array}\right) \\
& \sim 2^{-\rho} n^{\rho} \ell(n) \quad(n \rightarrow \infty),
\end{aligned}
$$

valid for each slowly varying function $\ell(\cdot)$ and $\rho \in R^{+}$.

Our second example is the classical Binomial Law, defined by

$$
P\left\{X_{n}=k\right\}=\left(\begin{array}{c}
n \\
k
\end{array}\right) p^{k}(1-p)^{n-k}, \quad 0<p<1,
$$

In this case $E X_{n}=n p ; \sigma^{2}\left(X_{n}\right)=n p(1-p)$. Hence, by Proposition 4 we get

$$
\sum_{k \leq n} k^{\rho} \ell(k)\left(\begin{array}{c}
n \\
k
\end{array}\right) p^{k}(1-p)^{n-k} \sim(n p)^{\rho} \ell(n p) \sim p^{\rho} n^{\rho} \ell(n)(n \rightarrow \infty) .
$$

for each slowly varying $\ell(\cdot)$ and $\rho \in R^{+}$.

\section{REFERENCES}

[1] M. Marden, Geometry of Polynomials, AMS: Providence, 1966.

[2] N.H. Bingham, C.M. Goldie, and J.I. Teugels, Regular Variation, Cambridge Univ. Press, 1989.

[3] Y.S. Chow and H. Teicher, Probability Theory, Berlin: SpringerVerlag, 1988.

[4] M. Vuilleumier, Sur le Comportement asymptotique des transformations linéaires des suites, Math. Zeitsch. vol. 98, pp. 126139, 1967.

[5] G. Szego, Orthogonal Polynomials. New York: AMS, 1959.

(C) Slavko Simic; Licensee Bentham Open.

This is an open access article licensed under the terms of the Creative Commons Attribution Non-Commercial License (http://creativecommons.org/licenses/by$\mathrm{nc} / 3.0 /$ ), which permits unrestricted, non-commercial use, distribution and reproduction in any medium, provided the work is properly cited. 